\title{
A new numerical approach to MHD flow of a Maxwell fluid past a vertical stretching sheet in the presence of thermophoresis and chemical reaction
}

\section{Stanford Shateyi*}

"Correspondence:

stanford.shateyi@univen.ac.za; sshateyi@yahoo.com

Department of Mathematics, University of Venda, P Bag X 5050 Thohoyandou, 0950, South Africa

\begin{abstract}
In this study we consider steady MHD flow of a Maxwell fluid past a vertical stretching sheet in a Darcian porous medium. The motion of the fluid is caused by the stretched sheet. The governing boundary layer equations for momentum, thermal energy and concentration are reduced using a similarity transformation to a set of coupled ordinary differential equations. The similarity ordinary differential equations are then solved numerically by a recently developed spectral relaxation method together with the Chebyshev pseudo-spectral collocation method. Effects of the physical parameters on the velocity, temperature and concentration profiles as well as the local skin friction coefficient and the heat and mass transfer rates are depicted graphically and/or in tabular form.
\end{abstract}

MSC: 65Pxx; 76-XX

Keywords: Maxwell fluid; stretching sheet; thermophoresis

\section{Introduction}

During the last few years, the boundary layer flows of non-Newtonian fluids driven by stretching surfaces have attracted much research interest [1-4]. This has been driven by their great importance in engineering and industrial applications. In particular, such applications are encountered in extrusion processes, glass-fiber and paper production, electronic chips, application of paints, food processing and movement of biological fluids. There is no single constitutive relationship between stress and rate of strain by which all the non-Newtonian fluids can be examined. The main difficulty in researching a general boundary-layer theory for non-Newtonian fluids lies, obviously, in the diversity of these fluids, in their constitutive behavior, simultaneous viscous and elastic properties such that differentiating between those effects which arise as a result of a fluid's shear-dependent viscosity from those which are attributable to the fluid's elasticity becomes virtually impossible. But some mathematical models have been proposed to fit well with the experimental observations [5]. The simplest model for the rheological effects of viscoelastic fluids is the Maxwell model where the dimensionless relaxation time is small. However, in some more concentrated polymeric fluids, the Maxwell model is also used for large dimensionless relaxation time. Vieru et al. [6] applied Fourie and Laplace transforms to find exact solutions of a fractional Maxwell model for flow between two-sided wall perpendicular

(c) 2013 Shateyi; licensee Springer. This is an Open Access article distributed under the terms of the Creative Commons Attribution License (http://creativecommons.org/licenses/by/2.0), which permits unrestricted use, distribution, and reproduction in any medium, provided the original work is properly cited. 
to a plate. Hayat et al. [7] analyzed the MHD flow and mass transfer of a UCM fluid past a porous shrinking sheet on the presence of chemical reaction species. Fetecau et al. [8, 9] investigated unsteady flows in Maxwell fluids with the flow being induced by oscillating/accelerated mature of the rigid body. Hayat et al. [10] studied the MHD unsteady flow of a Maxwell fluid in a rotating frame of reference and porous medium. It must be noted that the Maxwell fluid model allows for the relaxation effects which cannot be predicted in other different types of non-Newtonian fluids such as second, third and fourth grades.

The heat transfer analysis of boundary layer flow with radiation is very important in electrical power generation, astrophysical flows, solar power technology and space vehicle re-entry. Shateyi et al. [11] investigated the influence of a magnetic field on heat and mass transfer by mixed convection in the presence of Hall, radiation Soret and Dufour effects. Shateyi and Motsa [12] studied the effects of thermal radiation on heat and mass transfer over an unsteady stretching surface. Abel et al. [13] performed the analysis of the effect of MHD and thermal radiation on two-dimensional steady flow of an incompressible, upperconverted Maxwell fluid.

Thermophoresis is a mechanism of migration of small particles in the direction of a decreasing thermal gradient [14]. Thermophoresis causes small particles to deposit on the cold surfaces. It has many applications in aerosol technology, deposition of silicon thin films and radioactive particle deposition in nuclear reactor safety simulations. Tsai et al. [15] observed that the thermophoresis is an effective method for particle collection. The velocity acquired by the particle is called thermophoretic velocity, and the force experienced by the suspended particle is called thermophoretic force. Tsai and Huang [16] theoretically studied the steady stagnation point flow over a flat stretching surface in the presence of species concentration, and mass diffusion under Soret and Dufour effects was obtained by solving the governing equations of continuity, momentum, energy and concentration using similarity analysis and numerical technique.

Hayat and Qasim [17] investigated the MHD two-dimensional flow with heat and mass transfer over a stretching sheet in the presence of Joule heating and thermophoresis. Hayat et al. [18] studied three-dimensional flow of a non-Newtonian fluid induced by a stretching surface. Prasad et al. [19] investigated the effects of temperature-dependent viscosity, thermal conductivity and internal heat generation/absorption on the MHD flow and heat transfer of a non-Newtonian UCM fluid over a stretching sheet. Noor [20] considered the hydrodynamic flow of a Maxwell fluid past a vertical stretching sheet with thermophoresis. In this paper, the study of MHD flow of a Maxwell fluid with thermophoresis and chemical reaction, previously considered by Noor [20], will be investigated by using a recently developed numerical technique known as the spectral relaxation method. The method is based on simple iterative schemes which are formed by reducing the order of the momentum equation and by rearranging the resulting non-linear equations into a system of linear equations. The linear equations are then solved using the Chebyshev spectral collocation method [21, 22].

\section{Mathematical formulation}

We consider steady MHD flow of a Maxwell fluid past a vertical stretching sheet in a Darcian porous medium. The motion in the fluid is caused by the stretching of the sheet. A uniform magnetic field of strength $B_{0}$ is applied normally to the flow. We assume the magnetic Reynolds number to be sufficiently small so that the induced magnetic field can 
be neglected. At $t=0$, the sheet is impulsively stretched with the variable velocity $U_{w}(x)$; both the temperature distribution $T_{w}(x)$ and the concentration distribution $C_{w}(x)$ vary along the sheet. The fluid has uniform ambient temperature $T_{\infty}$ and uniform ambient concentration $C_{\infty}$ while $T_{w}>T_{\infty}$ and $C_{\infty}$. Under these assumptions and Boussineq's approximation, the governing equations for the model under consideration are written as

$$
\begin{aligned}
& \frac{\partial u}{\partial x}+\frac{\partial v}{\partial y}=0, \\
& u \frac{\partial u}{\partial x}+v \frac{\partial u}{\partial y}+\lambda_{1}\left[u^{2} \frac{\partial^{2} u}{\partial x^{2}}+v^{2} \frac{\partial^{2} u}{\partial y^{2}}+2 u v \frac{\partial^{2} u}{\partial x \partial y}\right] \\
& \quad=v \frac{\partial^{2} u}{\partial y^{2}}-\frac{\sigma B_{0}^{2}}{\rho}\left(u+\lambda_{1} v \frac{\partial u}{\partial y}\right)-\frac{v}{K} u+g \beta_{T}\left(T-T_{\infty}\right)+g \beta_{C}\left(C-C_{\infty}\right), \\
& u \frac{\partial T}{\partial x}+v \frac{\partial T}{\partial y}-\frac{\sigma B_{0}^{2}}{\rho c_{p}} u^{2}=\frac{\lambda_{g}}{\rho c_{p}} \frac{\partial^{2} T}{\partial y^{2}}+\frac{\mu}{\rho c_{p}}\left(\frac{\partial u}{\partial y}\right)^{2}-\frac{1}{\rho c_{p}} \frac{\partial q_{r}}{\partial y}, \\
& u \frac{\partial C}{\partial x}+v \frac{\partial C}{\partial y}=D \frac{\partial^{2} C}{\partial y^{2}}-\frac{\partial\left(V_{T} C\right)}{\partial y}-k_{2} C .
\end{aligned}
$$

The boundary conditions for this problem can be written as

$$
\begin{aligned}
& u(x, 0)=U_{w}(x)=a x, \quad v(x, 0)=0, \\
& T(x, 0)=T_{\infty}+b x, \quad C(x, 0)=C_{\infty}+c x, \\
& u(x, \infty)=0, \quad T(x, \infty)=T_{\infty}, \quad C(x, \infty)=C_{\infty},
\end{aligned}
$$

where $u$ and $v$ are the velocity components in the $x$-and $y$-directions, respectively, $v$ is the kinematic viscosity, $\rho$ is the fluid density, $K$ is the permeability of the porous medium, $\sigma$ is the electrical conductivity, $g$ is the acceleration due to gravity, $C$ is the fluid concentration, $T$ is the fluid temperature, $\beta_{T}$ and $\beta_{C}$ are the volumetric expansion coefficients of temperature and concentration, respectively, $\lambda_{g}$ is the fluid thermal conductivity, $c_{p}$ is the specific heat at constant pressure, $q_{r}$ is the radiative heat flux, $\mu$ is the dynamic viscosity, $D$ is the molecular diffusivity of the species concentration, $V_{T}$ is the thermophoretic velocity and $k_{2}$ is the chemical reaction parameter. By using the Rosseland diffusion approximation [23, 24] and following Raptis [25], among other researchers, the radiative heat flux, $q_{r}$ is given by

$$
q_{r}=-\frac{4 \sigma^{*}}{3 K_{s}} \frac{\partial T^{4}}{\partial y}
$$

where $\sigma^{*}$ and $K_{s}$ are the Stefan-Boltzman constant and the Rosseland mean absorption coefficient, respectively. We assume that the temperature differences within the flow are sufficiently small so that $T^{4}$ may be expressed as a linear function of temperature,

$$
T^{4} \approx 4 T_{\infty}^{3} T-3 T_{\infty}^{4}
$$

Using (7) and (8) in the last term of equation (3), we obtain

$$
\frac{\partial q_{r}}{\partial y}=-\frac{16 \sigma^{*} T_{\infty}^{3}}{3 K_{s}} \frac{\partial^{2} T}{\partial y^{2}}
$$


The thermophoretic velocity $V_{T}$, which appears in equation (4), can be written as

$$
V_{T}=-\frac{k v}{T_{r}} \frac{\partial T}{\partial y}
$$

where $T_{r}$ is the reference temperature and $k$ is the thermophoretic coefficient with a range of values from 0.2 to 1.2. We define a thermophoretic parameter $\tau$ as

$$
\tau=-\frac{k\left(T_{w}-T_{\infty}\right)}{T_{r}}
$$

\subsection{Similarity transformation}

The governing equations (2)-(4) can be transformed to a set of non-linear ordinary differential equations by introducing the following non-dimensional variables:

$$
\eta=\sqrt{\frac{a}{u}} y, \quad \psi=\sqrt{a \nu} x f(\eta), \quad \theta(\eta)=\frac{T-T_{\infty}}{T_{w}-T_{\infty}}, \quad \phi(\eta)=\frac{C-C_{\infty}}{C_{w}-C_{\infty}},
$$

where $\psi$ is the stream function that satisfies the continuity equation (1) with

$$
u=\frac{\partial \psi}{\partial y}=a x f^{\prime}(\eta), \quad v=-\frac{\partial \psi}{\partial x}=-\sqrt{a v} f(\eta)
$$

Using equations (12) and (13) in the governing equations, we obtain the following set of non-linear ordinary differential equations.

$$
\begin{aligned}
& f^{\prime \prime \prime}+(1+M \beta) f^{\prime \prime}-f^{\prime 2}+\beta\left(2 f^{\prime} f^{\prime \prime}-f^{2} f^{\prime \prime \prime}\right)-(\lambda+M) f^{\prime}+\gamma[\theta+N \phi]=0, \\
& \left(1+\frac{4}{3} R\right) \theta^{\prime \prime}+\operatorname{Pr}\left(f \theta^{\prime}-f^{\prime} \theta\right)+\operatorname{Pr} E c\left(M f^{\prime 2}+f^{\prime \prime 2}\right)=0, \\
& \phi^{\prime \prime}+S c\left[f \phi^{\prime}-f^{\prime} \phi-\tau\left(\theta^{\prime} \phi^{\prime}+\theta^{\prime \prime} \phi\right)\right]-K_{2} \phi=0 .
\end{aligned}
$$

Here primes denote differentiation with respect to $\eta, \beta=\lambda_{1} a$ is the Deborah number, $M=\frac{\sigma B_{0}^{2}}{\rho a}$ is the Hartmann number, $\lambda=\frac{v}{a K}$ is the porosity parameter, $\gamma=\frac{G r_{x}}{R e_{x}^{2}}$ is the local buoyancy parameter, $G r_{x}=\frac{g \beta_{T}\left(T_{w}-T_{\infty}\right) x^{3}}{v^{2}}$ is the Grashof number, $R e_{x}=\frac{U_{w} x}{v}$ is the Reynolds number, $N=\beta_{C}\left(C_{w}-C_{\infty}\right) /\left(\beta\left(T_{w}-T_{\infty}\right)\right)$ is the buoyancy ratio, $R=4 \sigma^{*} / K_{s} \lambda_{g}$ is the radiation parameter, $P r$ is the Prandtl number, $E c=U_{w}^{2} /\left(c_{p}\left(T_{w}-T_{\infty}\right)\right)$ is the Eckert number, $S c$ is the Schmidt number and $K_{2}=k_{2} S c / a$ is the chemical reaction. The boundary conditions are

$$
\begin{aligned}
& f(0)=0, \quad f^{\prime}(0)=1, \quad \theta(0)=1, \quad \phi(0)=1, \\
& f^{\prime} \rightarrow 0, \quad \theta \rightarrow 0, \quad \phi \rightarrow 0, \quad \text { as } \eta \rightarrow \infty .
\end{aligned}
$$

\section{Method of solution}

In this section we apply the proposed method of solution, hereinafter referred to as the spectral relaxation method (SRM), to solve the governing non-dimensional equations (14) to (17). The SRM method was recently developed by Motsa [26] and has been successfully used to solve a wide range of problems. The SRM algorithm starts with the assumption of 
having a system of $m$ non-linear ordinary differential equations in $m$ unknown functions $z_{i}(\eta), i=1,2, \ldots, m$, where $\eta \in[a, b]$ is the independent variable. The system of equations is then written in terms of $Z_{i}$ as a sum of its linear $\left(L_{i}\right)$ and non-linear components $\left(N_{i}\right)$ as indicated below.

$$
L_{i}\left[Z_{1}, Z_{2}, \ldots, Z_{m}\right]+N_{i}\left[Z_{i}, Z_{2}, \ldots, Z_{m}\right]=H_{i}(\eta), \quad i=1,2, \ldots, m
$$

where $H(\eta)$ is a known function of $\eta$. The non-linear components $\left(N_{i}\right)$ are then taken to the right-hand side of equation (19). The details of the SRM schemes are found in Motsa and Makukula [21]. We then use the Chebyshev pseudo-spectral method to solve the resultant iterative scheme. For details of the spectral methods, readers are referred to [27, 28 ]. Before applying the spectral method, the domain on which the governing equation is defined must be transformed to the interval $[-1,1]$, on which the spectral method can be implemented. We use the transformation $\eta=(b-a)(\tau+1) / 2$ to map the interval $[a, b]$ to $[-1,1]$.

Applying the SRM to (14) to (18), we first set $f^{\prime}(\eta)=g(\eta)$ and then write the equations as the following set of equations:

$$
\begin{aligned}
& f^{\prime}=g, \\
& g^{\prime \prime}+(1+\beta M) f g^{\prime}+\beta\left(2 f g g^{\prime}-f^{2} g^{\prime \prime}\right)-(\lambda+M) g-g^{2}-M g+\gamma(\theta+N \phi)=0, \\
& \left(1+\frac{4}{3} R\right) \theta^{\prime \prime}+\operatorname{Pr}\left(f \theta^{\prime}-g \theta\right)+\operatorname{Pr} E c\left(M g^{2}+g^{\prime 2}\right)=0, \\
& \phi^{\prime \prime}+S c\left[f \phi^{\prime}-g \phi-\tau\left(\theta^{\prime} \phi^{\prime}+\theta^{\prime \prime} \phi\right)\right]-K_{2} \phi=0,
\end{aligned}
$$

and the boundary conditions become

$$
\begin{aligned}
& f(0)=0, \quad g(0)=1, \quad \theta(0)=1, \quad \phi(0)=1, \\
& g(\infty)=0, \quad g^{\prime}(\infty)=0, \quad \theta(\infty)=0, \quad \phi(\infty)=0 .
\end{aligned}
$$

Equations (20) to (25) are then linearized to become

$$
\begin{aligned}
& f_{r+1}^{\prime}=g_{r}, \quad f_{r+1}(0)=0, \\
& g_{r+1}^{\prime \prime}+(1+\beta M) f_{r+1} g_{r+1}^{\prime}-(\lambda+M) g_{r+1}=g_{r}^{2}-\beta\left[2 f_{r+1} g_{r} g_{r}^{\prime}-f_{r+1}^{2} g_{r}^{\prime \prime}\right]-\gamma \theta_{r}-\gamma N \phi_{r}, \\
& g_{r+1}(0)=1, \quad g_{r+1}(\infty)=0, \\
& \frac{1}{P r}\left(1+\frac{4}{3} R\right) \theta_{r+1}^{\prime \prime}+f_{r+1} \theta_{r+1}^{\prime}-g_{r+1} \theta_{r+1}=-E c\left(M g_{r}^{2}+g_{r}^{\prime 2}\right), \\
& \theta_{r+1}(0)=1, \quad \theta_{r+1}(\infty)=0, \\
& \frac{1}{S c} \phi_{r+1}^{\prime \prime}+\left[f_{r+1} \phi_{r+1}^{\prime}-g_{r+1} \phi_{r+1}\right]-K_{2} \phi_{r}=\tau\left(\theta_{r}^{\prime} \phi_{r}^{\prime}+\theta_{r}^{\prime \prime} \phi_{r}\right), \\
& \phi_{r+1}(0)=1, \quad \phi_{r+1}(\infty)=0 .
\end{aligned}
$$

We note that the equations now form a system of linear decoupled equations which can be solved iteratively for $r=1,2, \ldots$, starting from initial guesses/approximations $\left(f_{0}(\eta), g_{0}(\eta), \theta_{0}(\eta), \phi_{0}(\eta)\right)$. 
Applying the Chebyshev pseudo-spectral method to (26) to (29), we obtain

$$
\begin{array}{lll}
A_{1} \mathbf{f}_{r+1}=B_{1}, & f_{r+1}\left(\tau_{\bar{N}}\right)=0, & \\
A_{2} \mathbf{g}_{r+1}=B_{2}, & g_{r+1}\left(\tau_{\bar{N}}\right)=1, & g_{r+1}\left(\tau_{0}\right)=0, \\
A_{3} \theta_{r+1}=B_{3}, & \theta_{r+1}\left(\tau_{\bar{N}}\right)=1, & \theta_{r+1}\left(\tau_{0}\right)=0, \\
A_{4} \phi_{r+1}=B_{4}, & \phi_{r+1}\left(\tau_{\bar{N}}\right)=1, & \phi_{r+1}\left(\tau_{0}\right)=0,
\end{array}
$$

where

$$
\begin{aligned}
& A_{1}=\mathbf{D}, \quad B_{1}=\mathbf{g}_{r} \\
& A_{2}=\mathbf{D}^{2}+\operatorname{diag}\left[(1+\beta M) \mathbf{f}_{r+1}\right] \mathbf{D}-(1+M) \mathbf{I}, \\
& B_{2}=\mathbf{g}_{r}^{2}-\beta\left[2 f_{r+1} g_{r} g_{r}^{\prime}-f_{r+1} g_{r}\right]-\gamma \theta_{r}-\gamma N \phi_{r}, \\
& A_{3}=\left(1+\frac{4 R}{3}\right) \mathbf{D}^{2}+\operatorname{diag}\left[\operatorname{Pr} \mathbf{f}_{r+1}\right] \mathbf{D}-\operatorname{diag}\left(\operatorname{Prg}_{r+1}\right),
\end{aligned}
$$

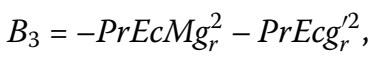

$$
\begin{aligned}
& A_{4}=\mathbf{D}^{2}+\operatorname{diag}\left[S c \mathbf{f}_{r+1}\right] \mathbf{D}-\operatorname{diag}\left(S c g_{r+1}+K_{2}\right), \\
& B_{4}=\operatorname{Sc\tau }\left[\theta_{r}^{\prime} \phi_{r}^{\prime}+\theta_{r}^{\prime \prime} \phi_{r}\right] \text {. }
\end{aligned}
$$

The initial approximation required to start the iterative process is

$$
\begin{array}{ll}
f_{0}=1-e^{-\eta}, & g_{0}(\eta)=e^{-\eta}, \\
\theta_{0}(\eta)=e^{-\eta}, & \phi_{0}(\eta)=e^{-\eta},
\end{array}
$$

which are convenient random functions that satisfy the boundary conditions. The iteration is repeated until convergence is achieved.

\section{Results and discussion}

In this section, we present the results obtained using the spectral relaxation method. The SRM method depends on the length of the governing domain $(b-a)$ and the number $(\bar{N})$ of collocation points. The SRM approach requires that we find the appropriate finite value $\eta_{\infty}$ which must be selected to be large enough to numerically approximate infinity and the behavior of the governing flow parameters at infinity. In order to select the appropriate value of $\eta$, we start with an initial guess, which is relatively small, and solve the governing SRM scheme equations over $\left[0, \eta_{\infty}\right]$ to obtain the solutions of flow parameters $f(\eta), g(\eta)$, $\theta(\eta)$ and $\phi \eta$. Once the optimal value of $\eta_{\infty}$ has been identified, we then use the same experiment to determine the minimum value of the number of grid points required to give solutions that do not depend on the grid size. In this study of the current problem, we used $\bar{N}=50, \eta_{\infty}=20$, with accuracy tolerance of $10^{-9}$. We also remark that convergence was achieved after as little as 10 iterations.

From the numerical computations, dimensionless velocity, temperature and concentration profiles as well as the local skin-friction coefficient, the local Nusselt number and the Sherwood number are presented for different values of the physical parameters significant in this study. The results are presented in tabular and graphical forms. Solutions 
are obtained for $\operatorname{Pr}=0.7 ; S c=0.5 ; N=1 ; \lambda=1 ; M=2 ; \gamma=1 ; R=0.3 ; K=1.0 ; \beta=0.2$; $\tau=0.2 ; E c=0.5$. In order to ascertain the accuracy and applicability as well as dependability of the current proposed newly developed method, the computed numerical values of the skin friction coefficient $-f^{\prime \prime}(0)$, the local Nusselt number $-\theta(0)$ and the Sherwood number $\phi(0)$ are compared with the previously generated results in [20] where the homotopy analysis method was used. These results are depicted in Tables 1-5. Though in [20]

Table 1 Comparison of the SRM results of $-f^{\prime \prime}(0),-\theta^{\prime}(0),-\phi^{\prime}(0)$ with those obtained in [20] for different values of the magnetic parameter

\begin{tabular}{|c|c|c|c|c|c|c|}
\hline \multirow[t]{2}{*}{$M$} & \multicolumn{2}{|l|}{$-f^{\prime \prime}(0)$} & \multicolumn{2}{|l|}{$-\theta^{\prime}(0)$} & \multicolumn{2}{|l|}{$-\phi^{\prime}(0)$} \\
\hline & {$[20]$} & Present & {$[20]$} & Present & {$[20]$} & Present \\
\hline$\overline{0.0}$ & 0.61105 & 0.61105243 & 0.63589 & 0.63588754 & 1.30284 & 1.30284353 \\
\hline 0.5 & 0.81242 & 0.81241682 & 0.53469 & 0.53469349 & 1.28395 & 1.28395421 \\
\hline 1.0 & 0.99660 & 0.99658887 & 0.44624 & 0.44621677 & 1.26728 & 1.26727829 \\
\hline 2.0 & 1.38934 & 1.32487675 & 0.28290 & 0.28719193 & 1.24527 & 1.2389943 \\
\hline
\end{tabular}

Table 2 Comparison of the SRM results of $-f^{\prime \prime}(0),-\theta^{\prime}(0),-\phi^{\prime}(0)$ with those obtained in [20] for different values of the chemical reaction parameter

\begin{tabular}{|c|c|c|c|c|c|c|}
\hline \multirow[t]{2}{*}{$K_{2}$} & \multicolumn{2}{|l|}{$-f^{\prime \prime}(0)$} & \multicolumn{2}{|l|}{$-\theta^{\prime}(0)$} & \multicolumn{2}{|l|}{$-\phi^{\prime}(0)$} \\
\hline & {$[20]$} & Present & {$[20]$} & Present & {$[20]$} & Present \\
\hline 0.0 & 0.92029 & 0.92027899 & 0.47847 & 0.47854048 & 0.70970 & 0.70959986 \\
\hline 0.5 & 0.96935 & 0.96934259 & 0.45647 & 0.45644780 & 1.03512 & 1.03512125 \\
\hline 1.0 & 0.99660 & 0.99658887 & 0.44624 & 0.44621677 & 1.26728 & 1.26727829 \\
\hline 2.0 & 1.03073 & 1.03072331 & 0.43520 & 0.43517486 & 1.62744 & 1.62744240 \\
\hline
\end{tabular}

Table 3 Comparison of the present results of $-f^{\prime \prime}(0),-\theta^{\prime}(0),-\phi^{\prime}(0)$ with those obtained in [20] as the thermophoresis parameter is varied

\begin{tabular}{|c|c|c|c|c|c|c|}
\hline \multirow[t]{2}{*}{$\tau$} & \multicolumn{2}{|l|}{$-f^{\prime \prime}(0)$} & \multicolumn{2}{|l|}{$-\theta^{\prime}(0)$} & \multicolumn{2}{|l|}{$-\phi^{\prime}(0)$} \\
\hline & {$[20]$} & Present & [20] & Present & [20] & Present \\
\hline 0.0 & 0.993696 & 0.99394752 & 0.44709 & 0.44706534 & 1.24182 & 1.24182644 \\
\hline 0.2 & 0.99660 & 0.996588887 & 0.44624 & 0.44621677 & 1.26728 & 1.26722829 \\
\hline 0.5 & 1.00052 & 1.00051054 & 0.44499 & 0.44496863 & 1.30586 & 1.30585794 \\
\hline 1.0 & 1.00695 & 1.00693761 & 0.44298 & 0.44295388 & 1.37122 & 1.37120617 \\
\hline
\end{tabular}

Table 4 Comparison of the SRM results of $-f^{\prime \prime}(0),-\theta(0),-\phi(0)$ with those obtained in [20] for different values of the porosity parameter

\begin{tabular}{|c|c|c|c|c|c|c|}
\hline \multirow[t]{2}{*}{$\lambda$} & \multicolumn{2}{|l|}{$-f^{\prime \prime}(0)$} & \multicolumn{2}{|l|}{$-\theta^{\prime}(0)$} & \multicolumn{2}{|l|}{$-\phi^{\prime}(0)$} \\
\hline & {$[20]$} & Present & {$[20]$} & Present & {$[20]$} & Present \\
\hline 0.0 & 0.61270 & 0.61270238 & 0.50658 & 0.50658616 & 1.24182 & 1.29851630 \\
\hline 0.5 & 0.81416 & 0.81416494 & 0.47655 & 0.47655131 & 1.26728 & 1.27936940 \\
\hline 1.0 & 0.99660 & 0.99658887 & 0.44624 & 0.44621677 & 1.30586 & 1.26727829 \\
\hline 2.0 & 1.34492 & 1.31831969 & 0.38715 & 0.38737709 & 1.37122 & 1.24647373 \\
\hline
\end{tabular}

Table 5 Effects of the Deborah number on the skin friction, heat flux and mass flux

\begin{tabular}{llll}
\hline $\boldsymbol{\beta}$ & $\boldsymbol{- f}^{\prime \prime}(\mathbf{0})$ & $\boldsymbol{\theta}^{\prime}(\mathbf{0})$ & $\boldsymbol{- \boldsymbol { \phi } ^ { \prime } ( \mathbf { 0 } )}$ \\
\hline 0.0 & 0.95042487 & 0.46037264 & 1.27219499 \\
0.5 & 1.06635679 & 0.42584920 & 1.26021407 \\
1.0 & 1.18203689 & 0.39446318 & 1.24938909 \\
2.0 & 1.29462059 & 0.36415504 & 1.239732147 \\
\hline
\end{tabular}


convergence was said to be achieved after 35 iterations, varying some parameters like the magnetic strength might affect that. In [20], accuracy was only up to $10^{-5}$, but in the current study, accuracy is up to $10^{-9}$, which we believe is good enough for any numerical method. In Table 1 we observe that for small values of the magnetic parameter, there is excellent agreement between our results and those of [20]. However, as $M$ becomes large, the previously obtained results become less accurate. From Table 1 , we observe that as the Hartmann number increases, the Lorentz drag force caused by electromagnetism increases, thereby causing the skin friction to increase. This, in turn, causes the thickness of the velocity boundary layer as well as the velocity to decrease as depicted in Figure 1 . With high values of the skin friction $\left(-f^{\prime \prime}(0)\right)$, more heat and concentration are absorbed, which results in less heat flux $-\theta^{\prime}(0)$ and mass flux $-\phi(0)$ as can be clearly seen in Table 1 .

In Table 2, we observe that there is an excellent agreement between our present results with those obtained by Noor [20] as the porosity of the medium is varied. The skin friction increases as the values of the porosity increase. The physical interpretation of this phenomenon is that imposing greater porosity on the medium causes a greater force in the opposite direction of the flow. The heat and mass fluxes are greatly reduced as values of the porosity increase.

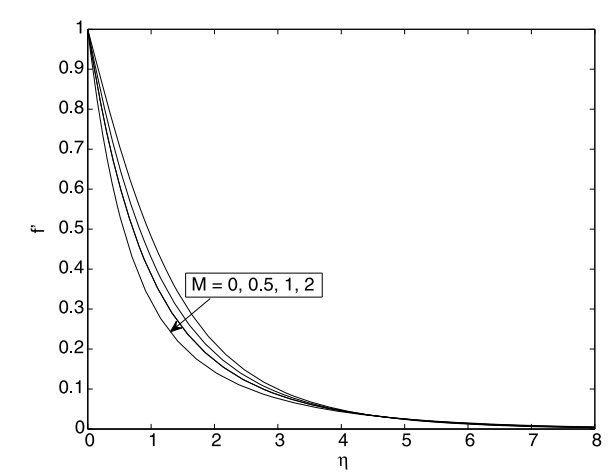

(a)

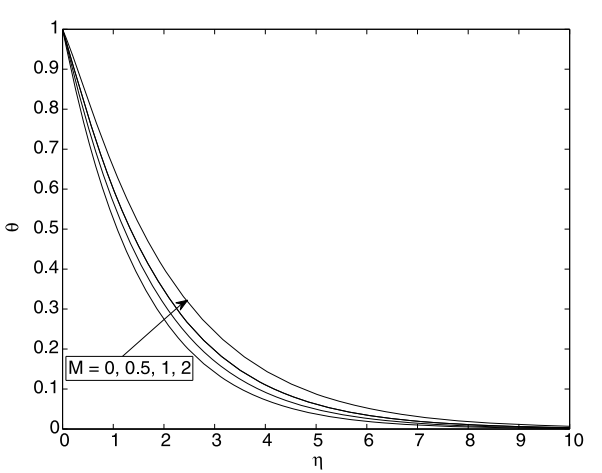

(b)

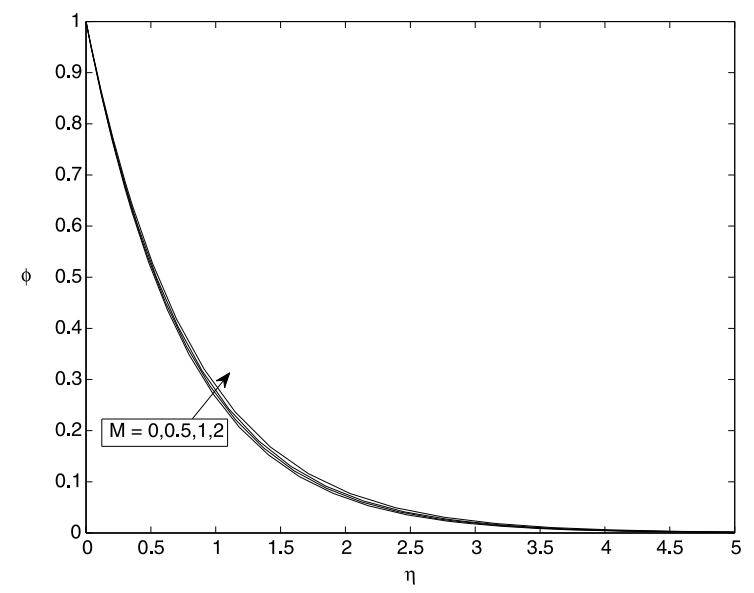

(c)

Figure 1 Variation of dimensionless (a) velocity, (b) temperature and (c) concentration profiles across the boundary layer for different values of $M$. 
The numerical results depicted in Tables 3 and 4 again show excellent agreement between the SRM and HAM results. In these tables, we observe consistent increments in the skin friction and mass flux but decrements in the heat flux as the thermophoresis and chemical reaction increase. We also observe in Table 3 that thermophoresis has more impact on the concentration profiles than on the velocity and temperature distributions. As the chemical reaction increases, higher fluid composition can be flushed away from the surface as shown in Table 4.

Table 5 depicts the influence of the Deborah number on the skin friction, heat and mass fluxes. As expected, increasing the Deborah number causes increments in the skin friction and decrements in the fluxes. Physically, at higher Deborah numbers, the material behavior changes to a non-Newtonian regime, increasingly dominated by elasticity, demonstrating solid-like behavior, hence high skin friction.

Figure 1(a)-(c) represents, respectively, the dimensionless velocity, temperature and concentration for various values of the magnetic field parameter $M$. From Figure 1(a), we observe that the velocity profiles are reduced as the magnetic parameter $M$ increases. Physically, increasing the magnetic field strength normal to the flow in an electrically conducting fluid produces a drag force known as the Lorentz force, which acts against the flow. Therefore, application of a moderate magnetic field normal to the flow can be used as a stabilizing mechanism, delaying the transition from laminar to turbulent flow. Reduction of the flow velocity due to the increase of the magnetic field strength causes increments in the heat and concentration profiles as depicted in Figure 1(b) and (c), respectively. Physically, applying the magnetic field heats up the fluid and thus reduces the heat and mass transfers from the wall causing increases in fluid temperature and concentration distributions.

In Figure 2(a)-(c), we display the influence of permeability of the medium on the flow velocity, temperature and concentration, respectively. We observe that the dimensionless velocity decreases as the values of the porosity parameter are increased. Physically, porosity allows more fluid to be taken away from the boundary layer and, therefore, reduces the velocity boundary layer but increases the thermal and solutal boundary layers as depicted in Figure 2(b) and (c).

Figure 3(a)-(b) shows the influence of a chemical reaction on the fluid velocity and concentration profiles. In this study, we are analyzing the effects of a destructive chemical reaction $\left(K_{2}>0\right)$. It is noticed that both velocity and concentration distributions decrease when the chemical reaction increases. Physically, for a destructive case, chemical reaction takes place with many disturbances. This, in turn, causes high molecular motion, which results in an increase in the transport phenomenon, thereby reducing the concentration distributions in the fluid flow.

The effects of the Deborah number $\beta$ on the velocity and temperature profiles are depicted in Figure 4(a)-(b), respectively. We observe that the boundary layer thickness decreases with increasing values of $\beta$ while the temperature increases. Physically, the Deborah number is the ratio of relaxation time characterizing the time it takes a material to adjust to applied stresses or deformations, and the characteristic time scale of an experiment probing the response of the material. At higher Deborah numbers, the material behavior changes to increasingly dominated by elasticity, demonstrating solid like behavior, thereby slowing down the flow velocity and increasing the temperature of the fluid. 


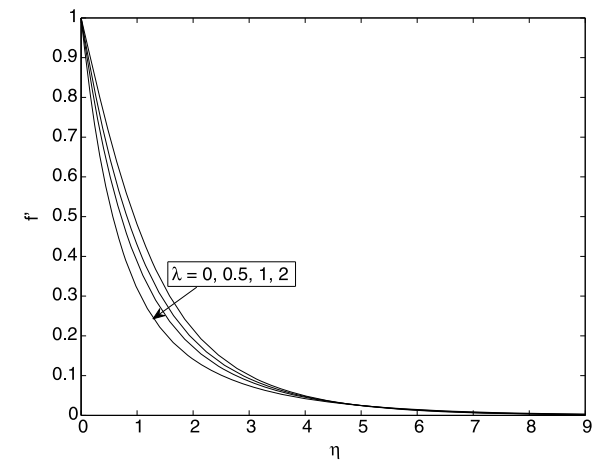

(a)

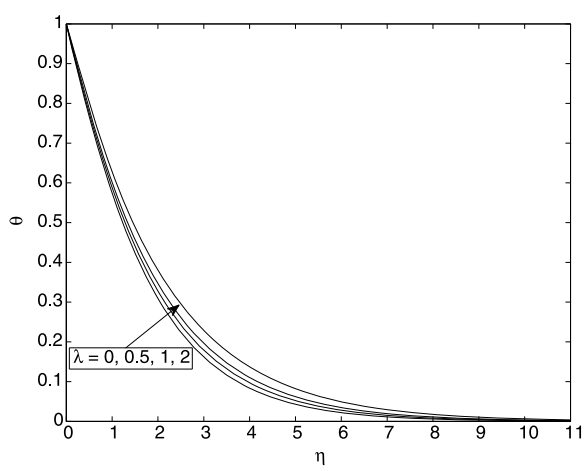

(b)

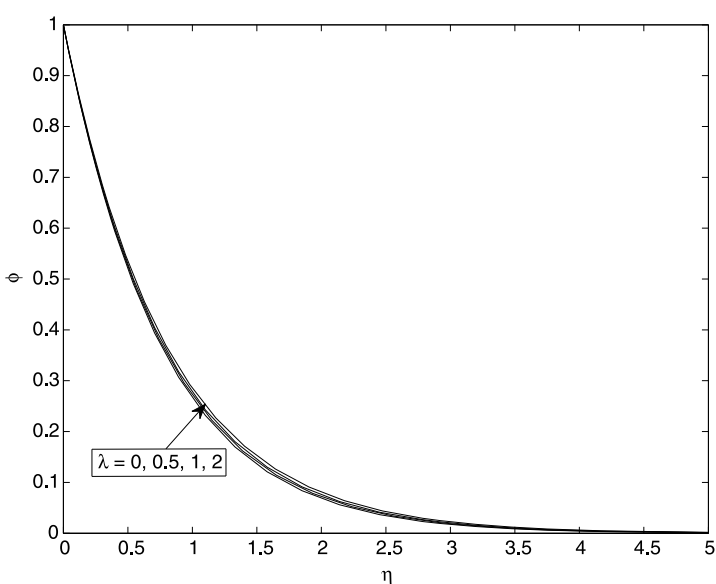

(c)

Figure 2 Effects of the porosity parameter on dimensionless (a) velocity, (b) temperature and (c) concentration profiles across the boundary layer.

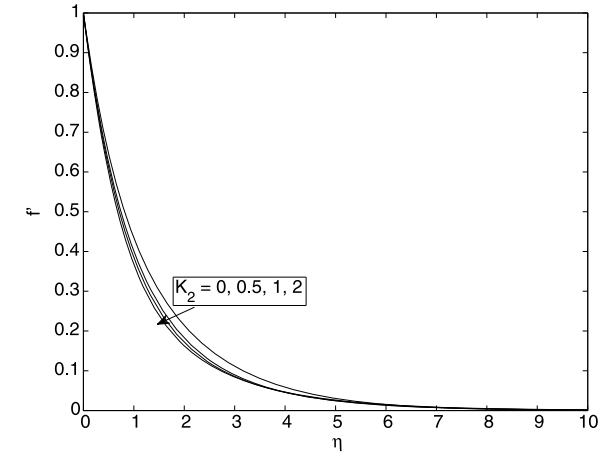

(a)

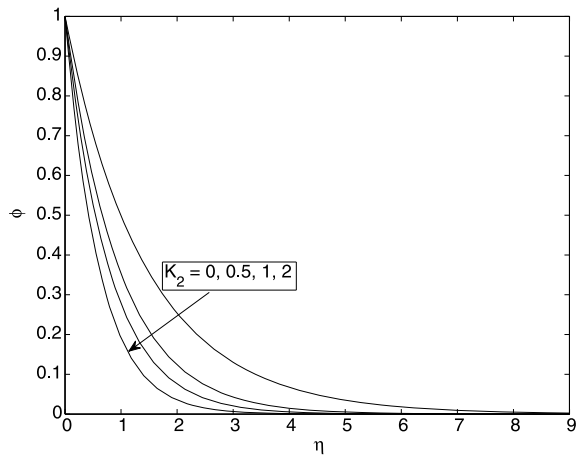

(b)

Figure 3 The influence of the chemical reaction parameter on dimensionless (a) velocity and (b) concentration profiles. 


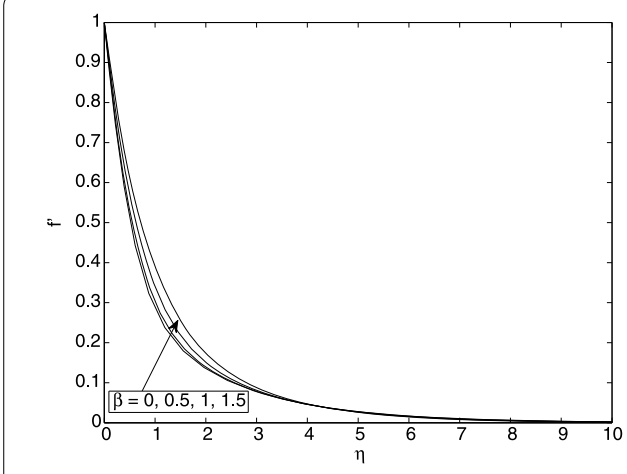

(a)

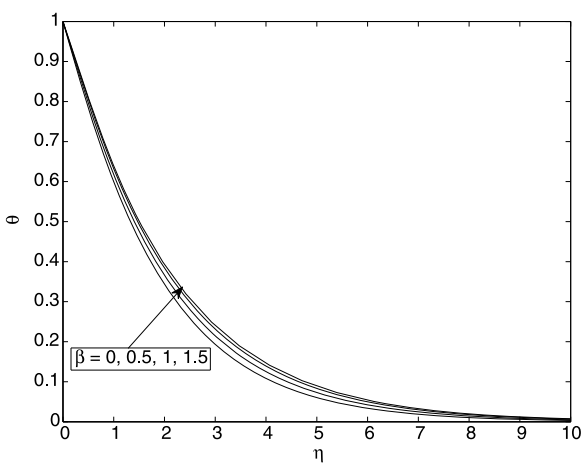

(b)

Figure 4 Variation of dimensionless (a) velocity and (b) temperature for different values of $\beta$.

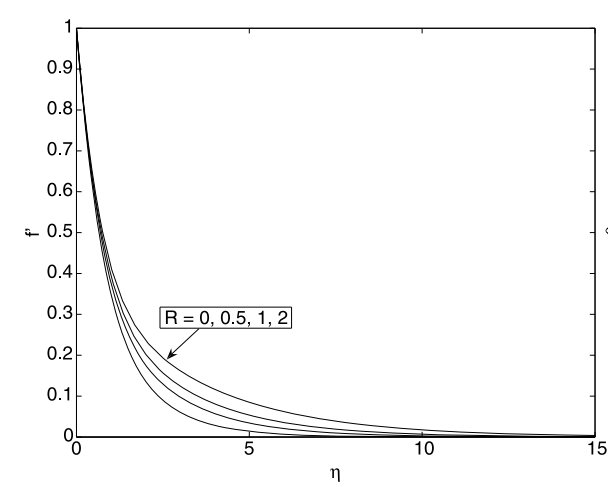

(a)

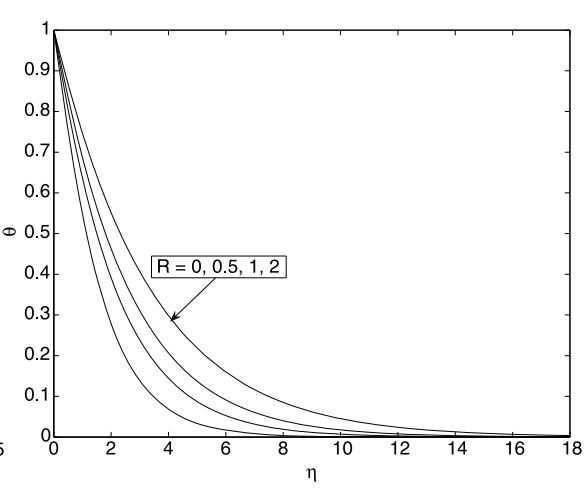

(b)

Figure 5 Variation of dimensionless (a) velocity and (b) temperature for different values of $R$.

The influence of the thermal radiation on fluid velocity and temperature is depicted in Figure 5(a)-(b), respectively. It is observed that the thermal radiation is to enhance fluid velocity in the boundary layer. It is interesting to note that thermal radiation has a significant influence on the temperature distribution within the fluid. We observe in Figure 5(b) that increasing thermal radiation causes the fluid temperature to increase. This is due to the fact that increase in the values of the thermal radiation parameter implies increasing radiation in the boundary layer, and hence increases the values of the temperature profiles in the thermal boundary layer.

Figure 6(a)-(b) represents the variation of velocity and temperature distributions in the boundary layer for various values of the Eckert number $E c$. We notice that the Eckert number has very little increasing effect on the velocity distribution in the boundary layer. By analyzing Figure 6(b), we observe that the effect of Eckert number is greatly increase the temperature in the flow region. This is because heat energy is stored in the liquid due to frictional heating. Thus the effect of increasing $E c$, is to enhance the temperature of the fluid.

Figure 7(a)-(b) depicts the effects of the Prandtl number on the velocity and temperature distributions. We observe that both velocity and temperature profiles are reduced as the Prandtl number increases. This is because when Pr increases, the thermal diffusiv- 


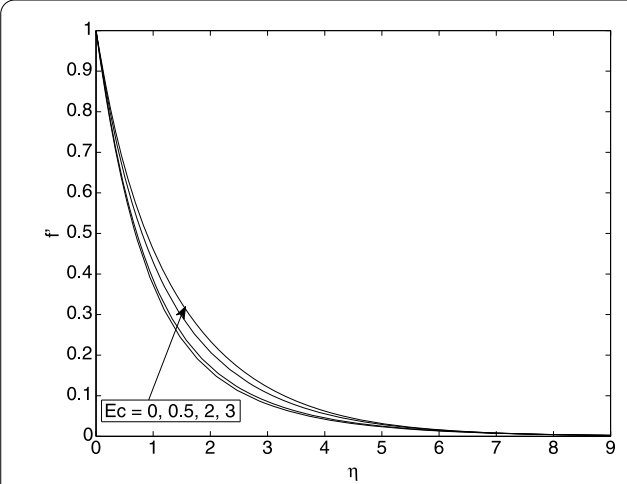

(a)

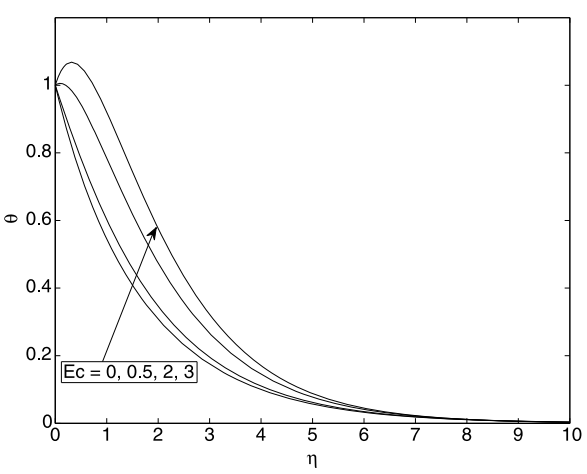

(b)

Figure 6 The influence of the Eckert number on the dimensionless (a) velocity and (b) temperature profiles.

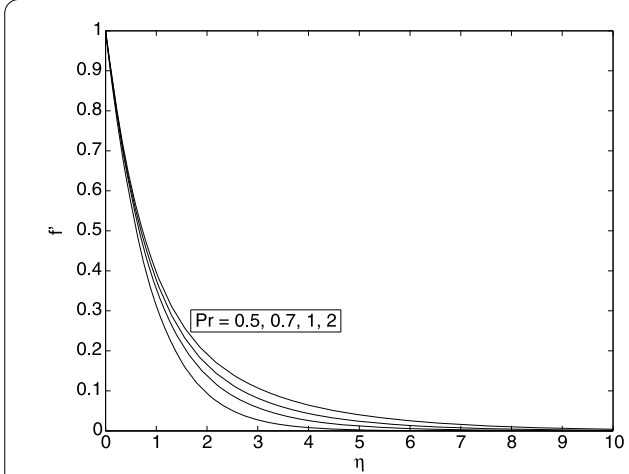

(a)

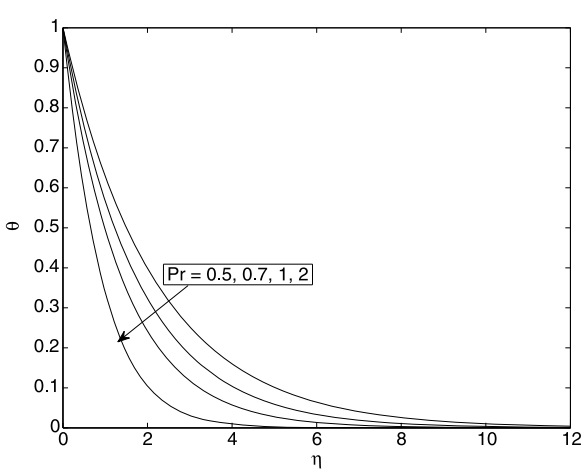

(b)

Figure 7 The effects of the Prandtl number on the dimensionless (a) velocity and (b) temperature profiles.

ity decreases leading to the decrease of energy transfer ability that decreases the thermal boundary layer.

Lastly, the effects of the thermophoretic parameter $\tau$ and the Schmidt number $S c$ are shown in Figure 8(a)-(b), respectively. We observe that increasing values of the thermophoretic parameter, $\tau$, induces a decrement in the concentration of the particles throughout the flow domain. We observe that the effect of increasing the thermophoretic parameter $\tau$ is limited to increasing slightly the wall slope of the concentration profiles but decreasing the concentration. The Schmidt number signifies the relative effectiveness of the momentum transport diffusion in the hydrodynamic boundary layer to the species diffusion in the concentration boundary layer. We clearly see from this figure that the concentration boundary layer thickness decreases as the Schmidt number $S c$ increases. This phenomenon occurs because when $S c$ increases, the concentration species becomes heavier.

\section{Conclusion}

A new numerical approach has been successfully used to solve the magnetohydrodynamic flow of a Maxwell fluid past a vertical stretching sheet in a Darcian porous medium under 


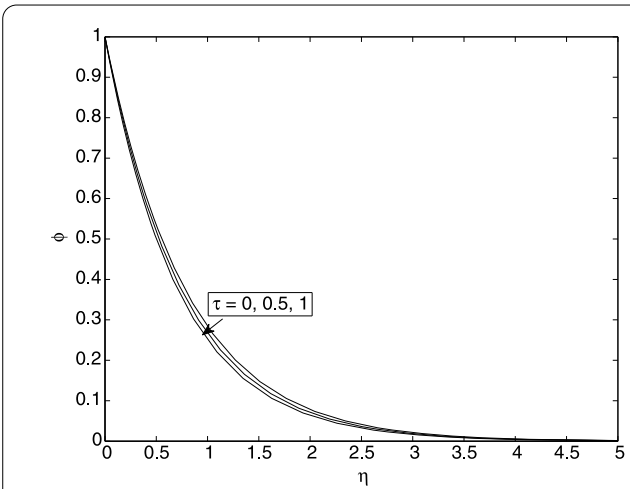

(a)

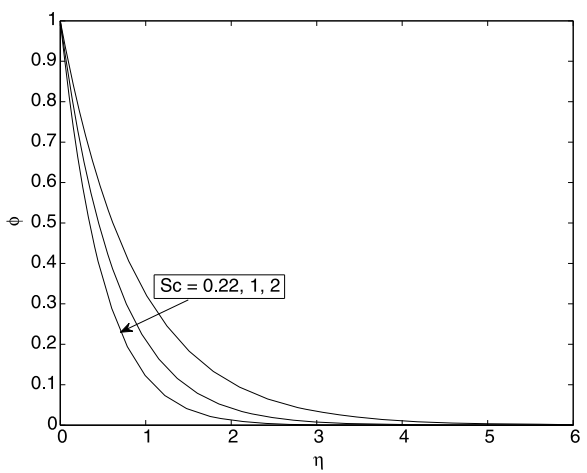

(b)

Figure 8 The effects of the thermophoretic parameter (a) and the Schmidt number (b) on the dimensionless concentration profiles.

the influence of thermophoresis, thermal radiation and a uniform chemical reaction. The investigation observed that this newly developed scheme had potential to be used even in complex non-linear problems. The velocity profiles were found to be greatly affected by magnetic strength, porosity, Deborah number as well as by thermal radiation. The fluid temperature was found to increase with increases in the magnetic strength, porosity, the Deborah number, thermal radiation as well as the Eckert number, but it decreased while the Prandtl number increased. The fluid concentration profiles were found to be significantly affected by the thermophoresis as well as by the presence of a chemical reaction.

Competing interests

The author declares that there is no conflict of interest in the use of the above mentioned software.

Author's contributions

This is a sole work carried out by SS.

\section{Acknowledgements}

The author wishes to acknowledge financial support from the University of Venda and NRF.

Received: 13 June 2013 Accepted: 12 August 2013 Published: 29 August 2013

\section{References}

1. Anderson, $\mathrm{HI}$, Hansen, $\mathrm{OR}$, Holmedal, B: Diffusion of a chemically reactive species from a stretching sheet. Int. J. Heat Mass Transf. 37(4), 659-664 (1994)

2. $\mathrm{Xu}, \mathrm{H}, \mathrm{Liao}, \mathrm{S}-\mathrm{J}$ : Laminar flow and heat transfer in the boundary-layer of non-Newtonian fluids over a stretching flat sheet. Comput. Math. Appl. 57, 1425-1431 (2009)

3. Mukhopadhyay, S: Casson fluid flow and heat transfer over a nonlinearly stretching surface. Chin. Phys. B 22(7), $074701(2013)$

4. Prasad, KV, Santhi, SR, Datti, PS: Non-Newtonian power-law fluid flow and heat transfer over a non-linearly stretching surface. Appl. Math. 3(5), 425-435 (2012)

5. Wu, J, Thompson, MC: Non-Newtonian shear-thinning flows past a flat plate. J. Non-Newton. Fluid Mech. 66, 127-144 (1996)

6. Vieru, D, Fetecau, C, Fetecau, C: Flow of a viscoelastic fluid with fractional Maxwell model between two side walls perpendicular to a plate. Appl. Math. Comput. 200, 459-464 (2008)

7. Hayat, T, Fetecau, C, Abbas, Z, Ali, N: Flow of a viscoelastic fluid with fractional Maxwell model between two side walls due to suddenly moved plate. Nonlinear Anal., Real World Appl. 9, 2288-2295 (2008)

8. Fetecau, C, Jamil, M, Fetecau, C, Siddique, I: A note on the second problem of Stokes for Maxwell fluids. Int. J. Non-Linear Mech. 44, 1085-1090 (2009)

9. Fetecau, C, Athar, M, Fetecau, C: Unsteady flow of generalized Maxwell fluid with fractional derivative due to a constantly accelerating plate. Comput. Math. Appl. 57, 596-603 (2009)

10. Hayat, T, Fetecau, C, Sajid, M: On MHD transient flow of a Maxwell fluid in a porous medium and rotating frame. Phys. Lett. A 372, 1639-1644 (2008)

11. Shateyi, S, Motsa, SS, Sibanda, P: The effects of thermal radiation, hall currents, Soret, and Dufour on MHD flow by mixed convection over a vertical surface in porous media. Math. Probl. Eng. 2010. Article ID 627475 (2010). doi:10.1155/2010/627475 
12. Shateyi, S, Motsa, SS: Thermal radiation effects on heat and mass transfer over an unsteady stretching surface. Math. Probl. Eng. 2009, Article ID 965603 (2009). doi:10.1155/2009/965603

13. Abel, MS, Tawade, JV, Shinde, JN: The effects of MHD flow and heat transfer for the UCM fluid over a stretching surface in presence of thermal radiation. Adv. Math. Phys. 2012, Article ID 702681 (2012). doi:10.1155/2012/702681

14. Hinds, WC: Aerosol Technology: Properties, Behavior, and Measurement of Airborne Particles. Wiley, New York (1982)

15. Tsai, C-J, Lin, J-S, Aggarwal, SG, Chen, D-R: Thermophoretic deposition of particles in laminar and turbulent tube flows. Aerosol Sci. Technol. 38, 131-139 (2004)

16. Tsai, R, Huang, JS: Heat and mass transfer for Soret and Dufour's effects on Hiemenz flow through porous medium onto a stretching surface. Int. J. Heat Mass Transf. 52, 2399-2406 (2009)

17. Hayat, T, Qasim, M: Influence of thermal radiation and Joule heating on MHD flow of a Maxwell fluid in the presence of thermophoresis. Int. J. Heat Mass Transf. 53, 4780-4788 (2010)

18. Hayat, T, Shehzad, SA, Alsaedi, A: Study on three-dimensional flow of Maxwell fluid over a stretching surface with convective boundary conditions. Int. J. Phys. Sci. 7(5), 761-768 (2012)

19. Prasad, KV, Sujatha, A, Vajravelu, K, Pop, I: MHD flow and heat transfer of a UCM fluid over a stretching surface with variable thermophysical properties. Meccanica 47, 1425-1439 (2012). doi:10.1007/s11012-011-9526-x

20. Noor, NFM: Analysis for MHD flow of a Maxwell fluid past a vertical stretching sheet in the presence of thermophoresis and chemical reaction. World Acad. Sci., Eng. Technol. 64, 1019-1023 (2012)

21. Motsa, SS, Makukula, ZG: On spectral relaxation method approach for steady von Kármán flow of a Reiner-Rivlin fluid with Joule heating and viscous dissipation. Cent. Eur. J. Phys. (2013). doi:10.2478/s11534-013-0182-8

22. Shateyi, S, Makinde, OD: Hydromagnetic stagnation-point flow towards a radially stretching convectively heated disk. Math. Probl. Eng. 2013, Article ID 616947 (2013). doi:10.1155/2013/616947

23. Hossain, MA, Alim, MA, Rees, DAS: The effect of radiation on free convection from a porous vertical plate. Int. J. Heat Mass Transf. 42(1), 181-191 (1999)

24. Seddeek, MA: Thermal radiation and buoyancy effects on MHD free convection heat generation flow over an accelerating permeable surface with temperature dependent viscosity. Can. J. Phys. 79, 725-732 (2001)

25. Raptis, A: Flow of a micropolar fluid past a continuously moving plate by the presence of radiation. Int. J. Heat Mass Transf. 41(18), 2865-2866 (1998)

26. Motsa, SS: New iterative methods for solving non-linear boundary value problems. In: Fifth Annual Workshop On Computational Applied Mathematics And Mathematical Modelling In Fluid Flow, Pietermaritzburg Campus, South Africa, 9-13 July (2012)

27. Canuto, C, Hussaini, MY, Quarteroni, A, Zang, TA: Spectral Methods in Fluid Dynamics. Springer, Berlin (1988)

28. Trefethen, LN: Spectral Methods in MATLAB. SIAM, Philadelphia (2000)

doi:10.1186/1687-2770-2013-196

Cite this article as: Shateyi: A new numerical approach to MHD flow of a Maxwell fluid past a vertical stretching sheet in the presence of thermophoresis and chemical reaction. Boundary Value Problems 2013 2013:196.

\section{Submit your manuscript to a SpringerOpen ${ }^{\odot}$ journal and benefit from:}

- Convenient online submission

- Rigorous peer review

- Immediate publication on acceptance

- Open access: articles freely available online

- High visibility within the field

- Retaining the copyright to your article 